\title{
High-Speed Bi-directional Optical Wireless System in Non-Directed Line-of-Sight Configuration
}

\author{
Giulio Cossu, Raffaele Corsini, and Ernesto Ciaramella, Senior Member, IEEE
}

\begin{abstract}
We propose and experimentally demonstrate the first bi-directional optical wireless link operating in non-direct line-ofsight (Nd-LOS). The system is based on visible light emitting diode (LED) for downlink and infrared LED for uplink. We achieve around $250 \mathrm{Mbit} / \mathrm{s}$ for both downstream and upstream transmission, with the ambient light noise of usual indoor illumination level (500 lx), produced by warm-white LED. $100 \mathrm{Mbit} / \mathrm{s}$ are guaranteed inside $12 \mathrm{~m}^{2}$ of a common room scenario, without LOS tracking. An optimized discrete multi-tone modulation gives bit-error-ratios always below of the common forward-error-correction threshold of $1.5 \times 10^{-3}$. These results indicate that this scheme has great potential for practical VLC system implementation.
\end{abstract}

Index Terms-Discrete multi-tone, light-emitting diodes, visible light communication.

\section{INTRODUCTION}

V ISIBLE light communication (VLC) can exploit light emitting diodes (LEDs) to provide both illumination and short-range wireless data transmission [1], [2]. Up to now, most of research activities were aimed at increasing the communication speed in order to demonstrate the high potential of the VLC systems [3], [4]. However these results can be only achieved under slightly unpractical conditions of short distance and very low mobility. Particularly, user-mobility would require a precise alignment, thus it needs a complex tracking. On the other hand, indoor optical wireless systems that do not require careful alignment thus allow user mobility, can be realized using either diffused non-line-of-sight (Dn-LOS) or non-directed LOS (NdLOS) configurations [5]. The first solution exploits the reflection of the light from the ceiling and can use a directional transmitter to establish the link. This architecture offers a higher link robustness, although it suffers from high optical losses due to the ceiling reflections and the longer path. Recently a Dn-LOS system has been reported [6] achieving $1 \mathrm{Gbit} / \mathrm{s}$ data rate, using a $50 \mathrm{~mW}$ infra-red (IR) laser diode at $808 \mathrm{~nm}$.

A good trade-off between robustness and link capacity is the Nd-LOS configuration, where wide-angle transmitters and receivers are employed. This architecture allows also the synergy

Manuscript received June 11, 2013; revised October 28, 2013 and January 26, 2014; accepted March 27, 2014. Date of publication April 3, 2014; date of current version May 12, 2014. This work was supported in part by the Tuscany Region under the project ARNO.

The authors are with the Scuola Superiore Sant'Anna, Pisa 56124, Italy, and also with CNIT Photonic Networks National Lab, 56124 Pisa, Italy (e-mail: g.cossu@sssup.it; r.corsini@sssup.it; e.ciaramella@sssup.it).

Color versions of one or more of the figures in this paper are available online at http://ieeexplore.ieee.org.

Digital Object Identifier 10.1109/JLT.2014.2315667 between illumination and data transmission, exploiting the LED sources for both purposes. To this aim, the receiver of a Nd-LOS system must have wide acceptance angle, thus it is also very sensitive to the ambient light, which is typically a combination of artificial illumination and sunlight. This can induce very high noise levels in the Nd-LOS VLC systems, thus strongly limiting the achievable data-rate [7].

For indoor VLC bidirectional communications, the realization of an uplink channel is still a challenging issue: as an example, a modulated retro-reflecting link was proposed in [8], which however achieved only low data rate because of the slow response of the modulators. Uplink using radio-frequency (RF) was also proposed [9], which however cannot be used in some RF-restricted areas, such as hospitals and/or airplanes. An optical wireless transmission using visible LED sources for both downlink and uplink was demonstrated in [10]. Although high-speed transmission was reported in that paper $(575 \mathrm{Mbit} / \mathrm{s}$ downlink and $225 \mathrm{Mbit} / \mathrm{s}$ uplink), it was achieved in unpractical conditions (distance of few centimeters and a focusing lens at the receivers). An optical bi-directional system was also described in [11], where a Nd-LOS visible light downlink and a directed LOS infrared uplink were established providing $100 \mathrm{Mbit} / \mathrm{s}$ transmission at about $3 \mathrm{~m}$ distance.

Recently, we presented preliminary results on a practical high-speed Nd-LOS VLC unidirectional system [12]. The system worked at typical distances of indoor environment (2-3 m) and provided simultaneously a high mobility and broadcast connectivity; although it exploited Nd-LOS configuration, it showed enhanced robustness to the ambient light. The effect of the background light on the system performance was deeply analyzed in order to optimize the system configuration. Namely, we found that the electrical spectrum of the noise due to the ambient light is mostly concentrated at low frequencies $(\ll 20 \mathrm{~Hz})$, thus it can be effectively suppressed by the high-pass electrical filter. Therefore, in our case the only significant effect of the ambient noise was to saturate the avalanche photo-diode (APD), reducing the amplitude of the receiver electrical signal [13]. In [12] we reduced the detrimental effect of the background light and improved the signal to noise ratio (SNR), by using an optical blue filter in front of the receiver [2].

In this paper we extend this analysis to achieve for the first time a bi-directional Nd-LOS system with high bit rate (around $250 \mathrm{Mbit} / \mathrm{s}$ ). We take as downstream a VLC system quite similar (yet improved) to the one introduced in [12] and also add an IR upstream communication link.

This paper is organized as follows. In Section II we present the experimental setup and discuss the key system parameters. In Section III we report the results of the measurements of 


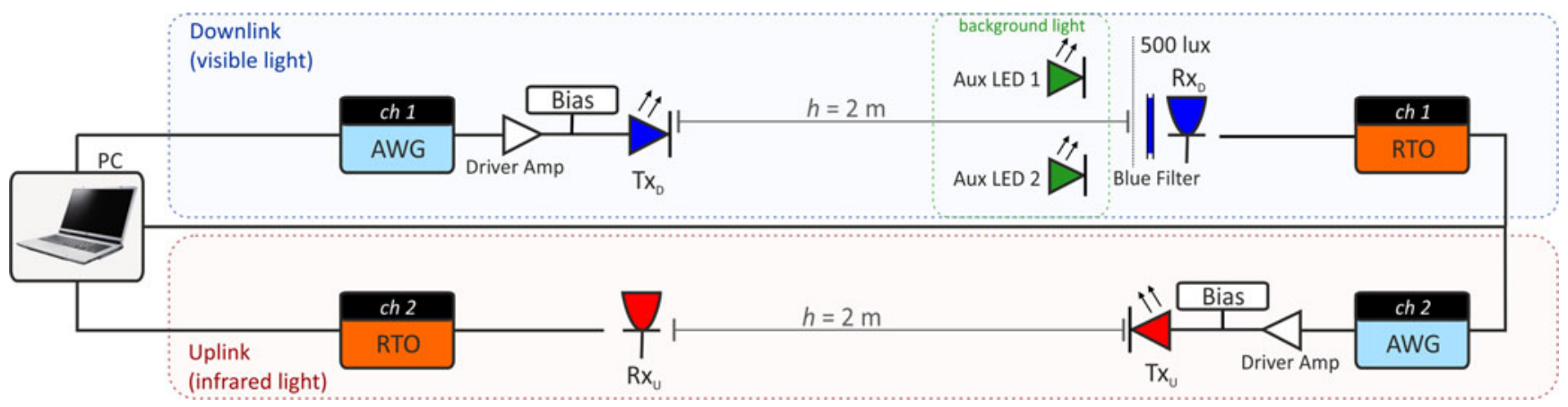

Fig. 1. Experimental setup of the bi-directional Non-directed-LOS VLC system. Tx $\mathrm{D}_{\mathrm{D}}$ : transmitter of downlink; $\mathrm{Tx}_{\mathrm{U}}$ : transmitter of uplink; $\mathrm{Rx}_{\mathrm{D}}$ : downlink receiver; $\mathrm{Rx}_{\mathrm{U}}$ : uplink receiver; Aux LEDs: optical sources for background light simulation; AWG: Arbitrary Waveform Generator; RTO: Real Time Oscilloscope.

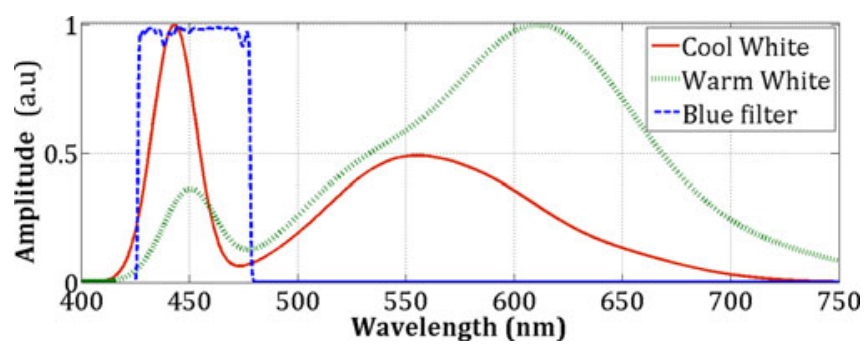

Fig. 2. Normalized optical spectra of the two visible LED sources used in the experiment. The plot shows also the transmittance of the blue filter in front of the receiver.

the crosstalk between the downstream and upstream channels. Then, in Section IV, we demonstrate a high speed transmission in typical practical conditions. The experiments were performed at realistic distance (from 2 to $2.8 \mathrm{~m}$ ), at the illuminance level common of office environment (500 lx [14]).

\section{EXPERIMENTAL SETUP}

We realized the experimental setup reported in Fig. 1. The downstream transmitter $\left(\mathrm{Tx}_{\mathrm{D}}\right)$ was a phosphorus cool-white LED assembly (color temperature $5650 \mathrm{~K}$, field-of-view, FOV, of $120^{\circ}$ ), including seven LED chips radiating a total flux of $1540 \mathrm{~lm}$ at bias current of $700 \mathrm{~mA}$. We simulated the ambient light at the downlink receiver $\left(\mathrm{Rx}_{\mathrm{D}}\right)$ by means of two auxiliary LEDs (Aux). The Aux LEDs were phosphorus warm white LEDs (color temperature $3000 \mathrm{~K}$, FOV of $120^{\circ}$ ), made of four chips radiating a total flux of $300 \mathrm{~lm}$ at bias current of $700 \mathrm{~mA}$. Thanks to the blue filter in front of the receiver, it allowed receiving the signal while rejecting most of the noise (the blue components of a warm-white LED are around $80 \%$ than in a cool-white LED). In this configuration, we measured that a common ambient light of 500 lx leads to a decrease of the SNR, which reduced around $15 \%$ the data rate of the multicarrier signal [12]. The normalized optical spectra of the different types of LED are reported in Fig. 2. As can be seen, they are fairly different, although providing similar white-light illumination.

The $\mathrm{Rx}_{\mathrm{D}}$ was an APD module consisting of an APD $\left(100 \mathrm{~mm}^{2}\right.$ active area, $11 \mathrm{MHz}$ high frequency cut off, $4 \mathrm{kHz}$ low frequency cut-off, responsivity peak at around $600 \mathrm{~nm}$ ) and an integrated Trans-Impedance Amplifier. Ignoring reflection losses, a bare detector achieves an effective area proportional to the cosine of the incident angle (Lambertian law). As explained before, we conveniently placed a blue filter in front of $\mathrm{Rx}_{\mathrm{D}}$, whose transmittance is also shown in Fig. 2; this was necessary to filter out the slow phosphorescent components of the $\mathrm{Tx}_{\mathrm{D}}$ and reject part of the ambient light generated by warm-white LEDs.

We drove $\mathrm{Tx}_{\mathrm{D}}$ by an optimized discrete multi-tone (DMT) signal, as described in [15]. The DMT waveform, consisting of $\mathrm{N}=280$ subcarriers in a bandwidth of $55 \mathrm{MHz}$, was generated offline and uploaded into the arbitrary waveform generator (AWG). The signal was first amplified by an electrical amplifier (25 dB gain, $29 \mathrm{dBm}$ minimum output power; $130 \mathrm{MHz} 3-\mathrm{dB}$ bandwidth), then superimposed via bias-T to the dc bias current (275 $\mathrm{mA}$ ) and eventually sent to the LED. After the transmission over the free-space, the APD output signal was sampled and stored by means of a real-time oscilloscope (RTO, $500 \mathrm{MSa} / \mathrm{s}$ ) for offline demodulation.

On the other side, an IR-LED (emitting at $850 \mathrm{~nm}$ with $30 \mathrm{~nm}$ full width at half-maximum, $3.6 \mathrm{~W}$ optical power at $1 \mathrm{~A}$ bias current, $120^{\circ} \mathrm{FOV}$ ) was used as uplink transmitter $\left(\mathrm{Tx}_{\mathrm{U}}\right)$. The assumption of an IR-LED for the uplink transmitter is common, as low-power infrared light could be placed very close to the user, provided that the eye safety conditions are fulfilled. The IR-LED used in this experiment is indeed compliant with the standards also at its maximum bias current (condition of the eye safety test).

An IR-LED source is completely exempt from risk, if the device that does not pose an IR radiation hazard for either the cornea nor for the retina, according to clause 4.3 of IEC 62471 [16]. Using the specifications provided by the datasheet at $1 \mathrm{~A}$ bias current, considering a realistic source-eye distance of $0.2 \mathrm{~m}$, the cornea exposure is found to be $30 \mathrm{Wm}^{-2}$, which is lower than the exposure limit fixed to $100 \mathrm{Wm}^{-2}$ for long exposure $(>1000 \mathrm{~s})$. We also calculated that the retinal exposure is $42 \mathrm{~mW} / \mathrm{mm}^{2} \mathrm{sr}$, which is lower than the retinal exposure limit (at $\lambda_{\mathrm{IR}}=850 \mathrm{~nm}$ ) of $60 \mathrm{~mW} / \mathrm{mm}^{2} \mathrm{sr}$ for weak visual stimulus (at $\lambda_{\mathrm{IR}}$ the pupil is fully opened). These values were obtained assuming $1 \mathrm{~A}$ bias current; however in our experiment, the bias current used for the communication was $250 \mathrm{~mA}$, which is much lower than the one used above. We thus conclude that the link emission is exempt from hazard both to cornea and retina, also for long exposures. 

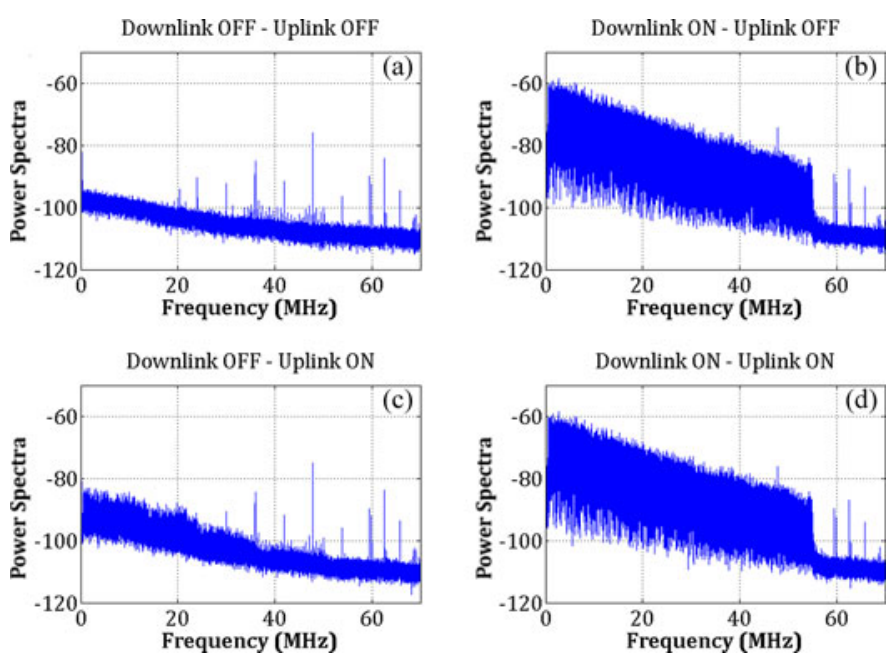

Fig. 3. (a)-(d) Downstream electrical spectra of the output of $\mathrm{Rx}_{\mathrm{D}}$ in all combination where downlink and uplink signal was turned on and off.

For the uplink, another DMT stream $(\mathrm{N}=256$ within $50 \mathrm{MHz}$ of bandwidth) was generated and uploaded to the second channel of the AWG. After an amplification stage (equal to the one used for downlink), we superimposed the DMT waveform to the dc bias current (250 mA) via bias-T. The signal was eventually received by an APD module $\left(19.6 \mathrm{~mm}^{2}\right.$ active area, $50 \mathrm{MHz}$ high frequency cut-off, responsivity peak at $800 \mathrm{~nm}$ ). No filter was placed in front of the receiver. The APD output was then stored by the RTO, for post-processing.

\section{CROSSTAlK CHARACTERIZATION}

In order to optimize the system, we first measured the electrical power spectra at $\mathrm{Rx}_{\mathrm{D}}$ output, in four different conditions obtained by switching downlink and uplink on and off. The downlink spectra are reported in Fig. 3. We first measured the noise of $\mathrm{Rx}_{\mathrm{D}}$ turning off all signals: as we see in Fig. 3(a), in the electrical spectrum several components were present. Although all these spurious frequencies were beyond the $3 \mathrm{~dB}$ bandwidth of the device some of them were within the spectral band of the DMT signal, which was indeed quite wider than the APD bandwidth (this can be seen in Fig. 3(b) where the spectrum of the downlink signal is reported).

We then turned on the uplink signal and switched off the downstream; the corresponding spectrum, which is reported in Fig. 3(c), shows higher noise in the range $<40 \mathrm{MHz}$. This effect was ascribed to electromagnetic interference (EMI) of the electrical signal irradiated by the IR-LED source and received by $\mathrm{Rx}_{\mathrm{D}}$, placed next to each other. A reduction of the EMI was indeed observed if the modules were moved away one from each other. We did not expect any optical crosstalk because of the blue filter in front of $\mathrm{Rx}_{\mathrm{D}}$, which removed all infrared light $\left(<10^{-3}\right.$ transmittance amplitude @ $\left.850 \mathrm{~nm}\right)$. In order to experimentally confirm, we placed an opaque screen in front of $\mathrm{Rx}_{\mathrm{D}}$ which removed completely the optical crosstalk from the IR-LED: indeed, in that case, no change of the noise level was observed. In Fig. 3(d) is reported the $\mathrm{Rx}_{\mathrm{D}}$ output electrical spectrum when both downlink and uplink signal were on.
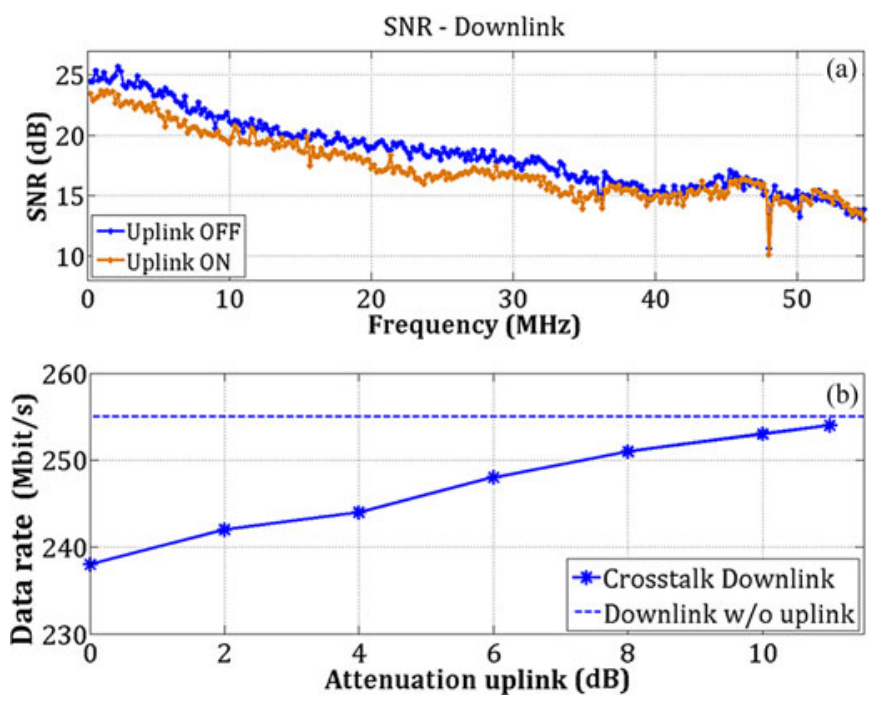

Fig. 4. (a) Measured SNR without (blue curve) and with the uplink signal on (red curve). (b) Download data-rate varying by the electrical power of the uplink signal at $\mathrm{BER}<2 \cdot 10^{-3}$.

The effect of crosstalk on the DMT signal can be seen in Fig. 4(a), where we reported the SNR of the downlink, without (blue line) and with (orange line) the presence of the uplink. Here, the channel frequency response was estimated by using a DMT signal probe where all the subcarriers were modulated with a BPSK having equal power. The SNR was then estimated from the error vector magnitude (EVM) of the received constellations [18]. The SNR drops around $2 \mathrm{~dB}$ below $40 \mathrm{MHz}$ due to the EMI from uplink transmitter. In the same plot, we see the effect of the spurious component at $47.8 \mathrm{MHz}$, where one of the noise peaks is located, as a dip in the SNR curve. The total electrical crosstalk introduced a decrease of data rate of $17 \mathrm{Mbit} / \mathrm{s}$ from the condition where the uplink was turned off [dotted line in Fig. 4(b)]. This reduced down to a negligible data-rate penalty ( $-1 \mathrm{Mbit} / \mathrm{s})$, when attenuating the uplink electrical power by $11 \mathrm{~dB}$ [solid line in Fig. 4(b)].

The same analysis was performed for the uplink channel. The electrical spectra of the uplink received signal are shown in Fig. 5(a)-(d). In this case spurious frequency peaks were concentrated at low frequency $[<2.5 \mathrm{MHz}$, Fig. 5(a)]. This difference compared to the $\mathrm{Rx}_{\mathrm{D}}$ [Fig. 3(a)] comes from a different APD module used for upstream, with higher responsivity in the IR region than the APD in $R x_{D}$.

We then turned on the downlink channel [Fig. 5(b)], and observed that some crosstalk components arose in the spectrum. A portion of this crosstalk was within the signal bandwidth which extends up to $50 \mathrm{MHz}$, as reported in Fig. 5(c). The low frequency crosstalk components was due to optical crosstalk, since the $\mathrm{Rx}_{U}$ was also sensitive to visible light and no IR filter was placed to filter out the visible light. In order to proof this, we used an opaque screen in front of $\mathrm{Rx}_{U}$ which removed completely the $\mathrm{Tx}_{\mathrm{D}}$ visible light signal: in this case, the noise at low frequency was not present in the spectrum. In the frequency range between 40 and $55 \mathrm{MHz}$, low EMI was present due to close proximity between $\mathrm{Tx}_{\mathrm{D}}$ and $\mathrm{Rx}_{\mathrm{U}}$. 

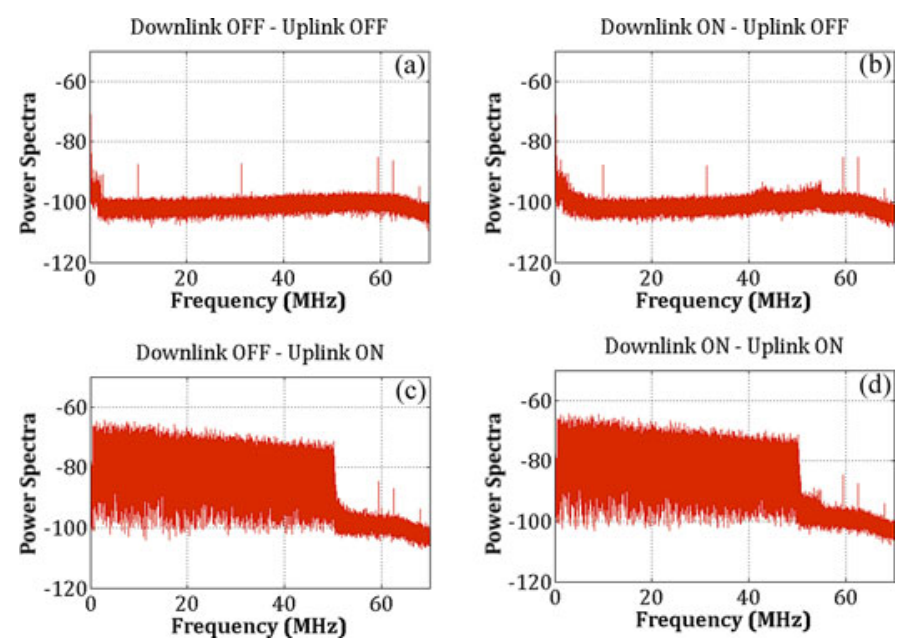

Fig. 5. (a)-(d) Upstream electrical spectra of the output of RxU in all combination where downlink and uplink signal was turned on and off.
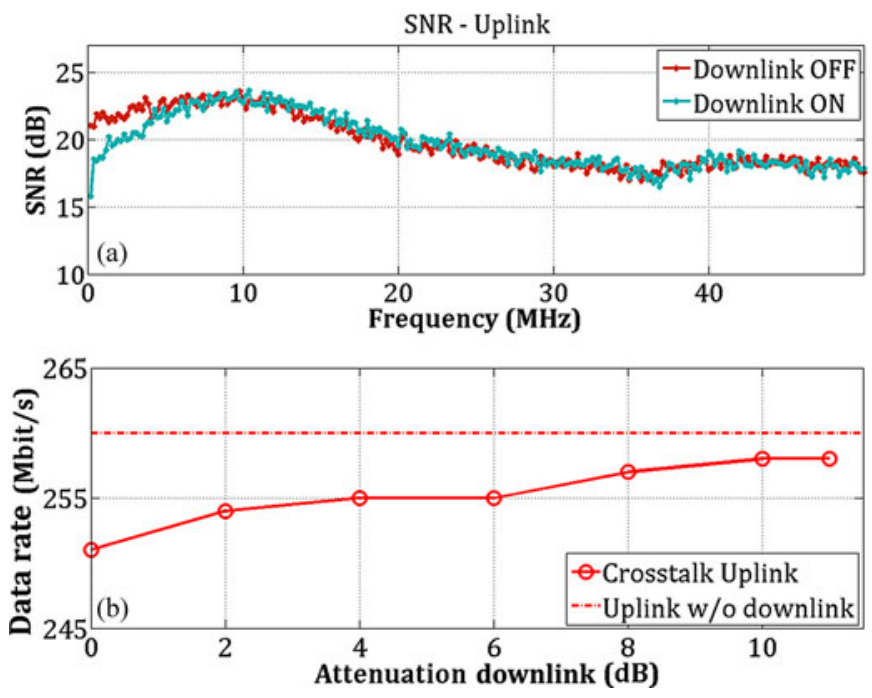

Fig. 6. (a) Measured SNR before and after turning on the downlink signal. (b) Uplink data-rate varying the electrical power of the downlink signal at BER $<2 \cdot 10^{-3}$.

As can be seen in Fig. 6(a), the above optical crosstalk had an impact on the SNR curve. The SNR dropped by maximum $5 \mathrm{~dB}$ in the low-frequency region, while at high frequency no significant effect was noticed. This is due to the low power of the EMI and because part of it was outside the signal band [from 50 to $55 \mathrm{MHz}$, as can be seen from Fig. 5(d)]. The total crosstalk reduced the data-rate by a limited amount $(9 \mathrm{Mbit} / \mathrm{s})$, from the condition without the downlink [dotted line in Fig. 6(b)]. This penalty could be further reduced to $1 \mathrm{Mbit} / \mathrm{s}$, by attenuating the downlink electrical power by $11 \mathrm{~dB}$ [solid line in Fig. 6(b)].

\section{TRANSMISSION EXPERIMENT}

Once the crosstalk was characterized, a bi-directional NdLOS transmission system could be finally demonstrated. The link is sketched in Fig. 7(a). In order to emulate a realistic scenario, we considered a vertical distance $h$ between the $\mathrm{Tx}_{\mathrm{D}}$ and the $\mathrm{Rx}_{\mathrm{D}}$ plane of $2 \mathrm{~m}$. The $\mathrm{Rx}_{\mathrm{D}}$ alignment condition is
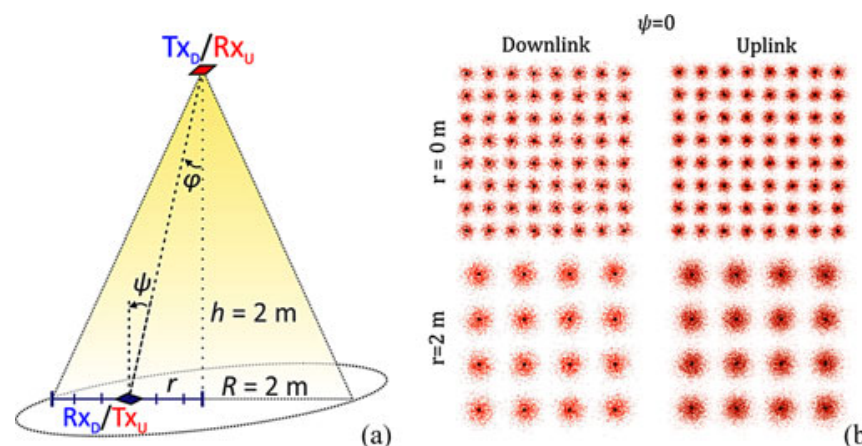

(b)

Fig. 7. (a) Schematic representation of bi-directional Nd-LOS scenario. (b) Constellation diagrams of the highest modulation loading for best condition ( $\mathrm{r}=0 \mathrm{~m}, \psi=0$, first line) and $\mathrm{r}=2$ with $\psi=\varphi$ (second line). The constellation are taken for both link (downstream: first column; upstream: second column). Goodness of fit: $99 \%$ for all the constellation diagrams.

identified by two angles: $\varphi$ is the angle between the normal of $\mathrm{Tx}_{\mathrm{D}}$ and the LOS with the $\mathrm{Rx}_{\mathrm{D}}$ and $\psi$ is the pointing angle of the $\mathrm{Rx}_{\mathrm{D}}$. Perfect LOS is achieved setting $\psi=0$. When $\psi=$ $\varphi$, the $\mathrm{Rx}_{\mathrm{D}}$ does not track the $\mathrm{Tx}_{\mathrm{D}}$ position, i.e. points always upward the ceiling. The same considerations can be made also for the uplink, where the $\mathrm{Tx}_{\mathrm{U}}$ was moved together with the $\mathrm{Rx}_{\mathrm{D}}$, whilst the $\mathrm{Rx}_{\mathrm{U}}$ was kept fixed in the ceiling pointing downward.

We measured the system performance for both downlink and uplink by varying the radial distance $r$ from 0 to $2 \mathrm{~m}$ : at each distance both of the above conditions $(\psi=\varphi$ and $\psi=0$ ) were considered. The signal illuminance range from 30 to $10 \mathrm{~lx}$ when the $\mathrm{Rx}_{\mathrm{D}}$ was moved from $r=0 \mathrm{~m}$ to $r=2 \mathrm{~m}$, whilst Aux LEDs allowed us to generate an ambient light giving $500 \mathrm{~lx}$ at the $\mathrm{Rx}_{\mathrm{D}}$.

In Fig. 7(b), we report four constellation diagrams for the carriers having highest modulation order with $\psi=0$ at $r=0 \mathrm{~m}$ (first line) and $r=2 \mathrm{~m}$ (second line). Both downlink (first column) and uplink (second column) constellations are reported. Clearly, when increasing the distance, the modulation order was decreased adapting to the lower SNR.

The measured data-rate and the received optical power vs. radial distance $r$ are summarized in Fig. 8 (downlink) and Fig. 9 (uplink), where the solid lines report the data rate obtained at $\mathrm{BER}<1.5 \times 10^{-3}$ with $\psi=0$ (best pointing condition), while the dashed lines show the data-rate with $\psi=\varphi$ (vertical alignment). This BER value is the upper limit for common forward error correction (FEC) [19] and is required for good performance in common Radio Frequency wireless systems. Together with the data rate, in the same figures we report the received optical power. We always obtained a minimum of $100 \mathrm{Mbit} / \mathrm{s}$ in the hot spot area having $r \leq 2 \mathrm{~m}\left(\approx 12 \mathrm{~m}^{2}\right.$ area). This value is compliant with the standard HD-video transmission [20]. A maximum of $238 \mathrm{Mbit} / \mathrm{s}$ was achieved in the best configuration. Similar results were obtained for uplink transmission where a maximum of $255 \mathrm{Mbit} / \mathrm{s}$ in perfect alignment and a guarantee speed of $100 \mathrm{Mbit} / \mathrm{s}$ in the vertical alignment were achieved. Therefore the system shows a high robustness also to the pointing angle of the receiver.

Finally, we checked the Gaussian distribution of the noise; as it is well known, this is a key feature that must be checked 

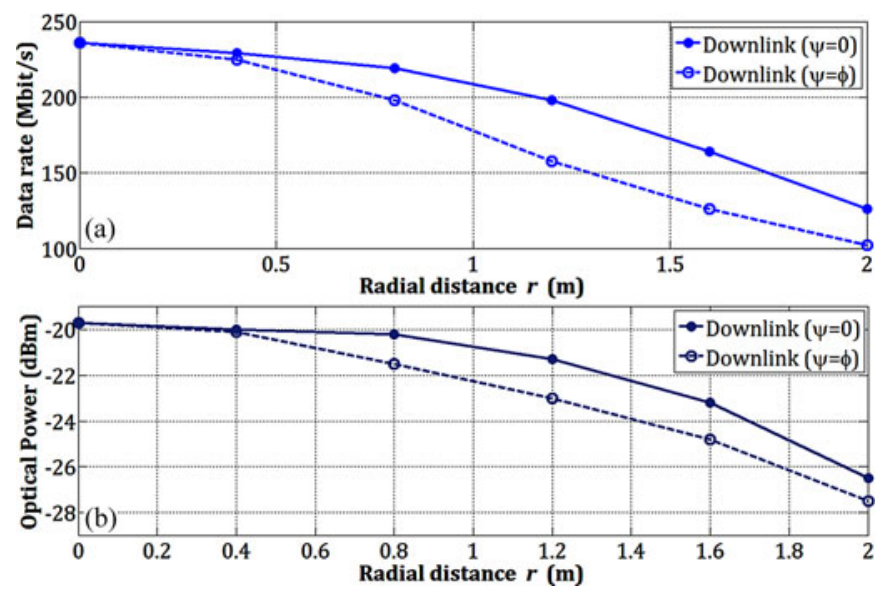

Fig. 8. (a) Downlink data-rate vs. radial distance $r$, for either best pointing condition $(\psi=0)$ and vertical alignment $(\psi=\varphi)$. (b) Received downlink optical power as a function of the radial distance.
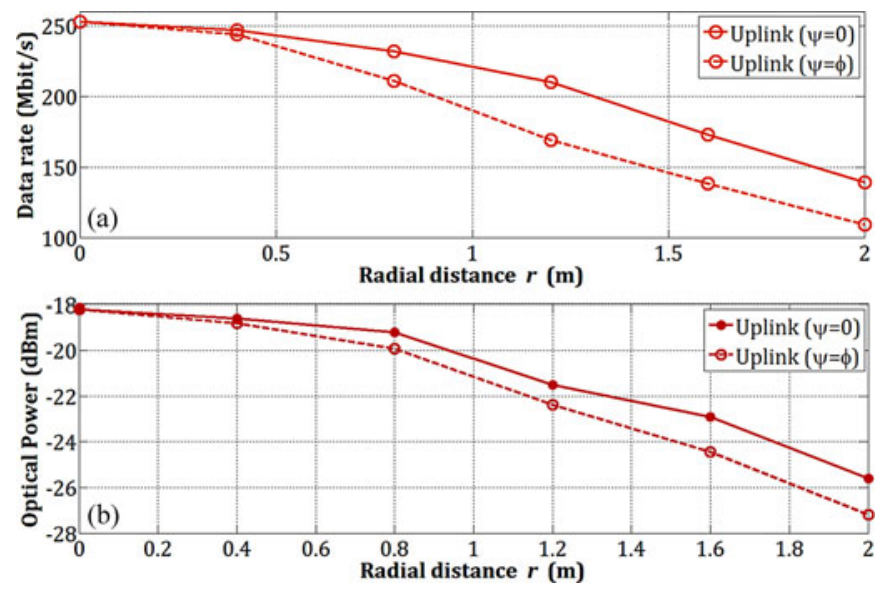

Fig. 9. (a) Uplink data-rate vs. radial distance $r$, for either best pointing condition $(\psi=0)$ and vertical alignment $(\psi=\varphi)$. (b) Received uplink optical power as a function of the radial distance.

in order to exploit FEC which can indeed provide optimal BER improvements only if the statistical distribution of the noise is Gaussian.

Therefore we first estimated the BER values as a function of the subcarrier index, both for downlink and uplink in the best condition $(r=0)$. The BER values, which are reported in Fig. 10, were estimated from the theoretical relationship between error vector magnitude (EVM) and BER [18]. This relationship is based on two assumptions: Additive White Gaussian Noise and the number of symbols in every subcarrier much higher than the number of distinct constellation points in that subcarrier [18]. Both conditions were met in our case. Indeed the goodnessof-fit values [reported in Fig. 7(b)] for the 2-D Gaussian fit of the EVM in the received constellations was always greater than $99 \%$, indicating that the noise had a Gaussian distribution; furthermore we sent 7500 symbols per carrier and the maximum number of distinct constellation points was 64 .

The estimated total BER for downlink (uplink), defined as the average BER values over all the subcarriers, was $1.5 \times 10^{-3}$ $\left(1.9 \times 10^{-3}\right)$. The experimental BER (measured after offline

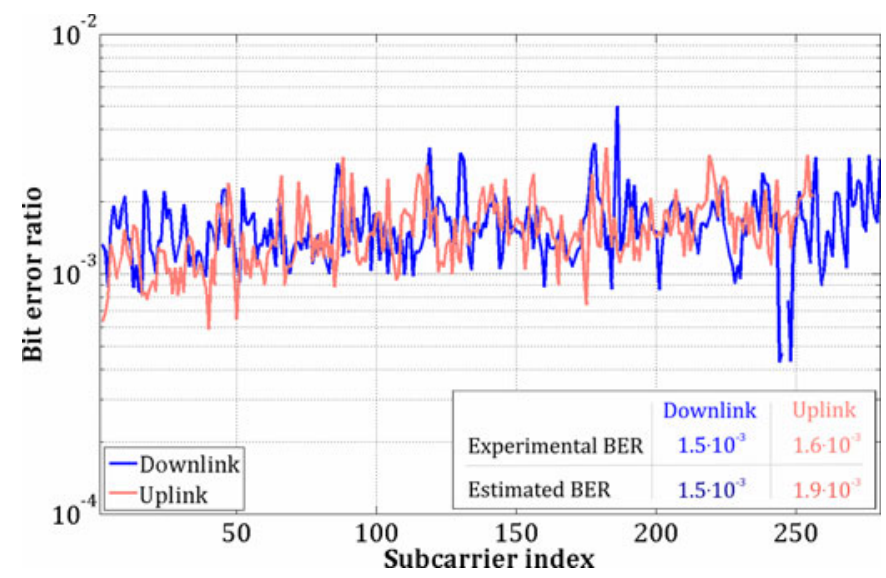

Fig. 10. Estimated BER vs subcarrier index for both links in best condition $(r=0$ and $\psi=0)$.

demodulation of DMT frames composed of approx. 10 million bits) was $1.5 \times 10^{3}\left(1.6 \times 10^{-3}\right)$ for downlink (uplink). The estimated and the experimental BER values were consistent, thus demonstrating that the transmission has not suffered from non-linear effects, which may arise from the transmitter devices (LEDs and amplifier) [15].

\section{CONCLUSION}

Using common LEDs as transmitters, we demonstrated the first practical high-speed bi-directional optical wireless transmission operating in Nd-LOS configuration in a typical indoor scenario, i.e. at an ambient light (generated by warm-white LEDs) level of $500 \mathrm{~lx}$, and at a realistic distance of $2 \mathrm{~m}$ (with 30 lux signal). The optical wireless system exploited a visible LED as optical source for the downstream and IR-LED for upstream.

After a detailed analysis of the crosstalk between the downlink and uplink channels, we characterized the system obtaining values of the data-rate between 100 and $250 \mathrm{Mbit} / \mathrm{s}$ (BER lower than the FEC limit, $1.5 \times 10^{-3}$ ) in the considered practical range of conditions. These high data rates were achieved by efficiently exploiting the small LED bandwidth due to a combination of DMT, multi-level modulation and bit/power-loading. A key role was also played by the optical filter at the Rx.

Furthermore, we demonstrated a high angle tolerance at the receiver allowing high mobility of the user. These results represent an important step towards practical implementations of the optical wireless systems for indoor environments. Further improvements are expected by a refined choice of the Tx LED and of the Rx.

\section{REFERENCES}

[1] M. Kavehrad, "Sustainable energy-efficient wireless applications using light," IEEE Commun. Mag., vol. 48, no. 12, pp. 66-73, Dec. 2010.

[2] D. C. O'Brien, L. Zeng, H. Le-Minh, G. Faulkner, J. W. Walewski, and S. Randel, "Visible light communications: Challenges and possibilities," in Proc. IEEE 19th Int. Symp., Sep. 15-18, 2008, pp. 1-5.

[3] G. Cossu, A. M. Khalid, P. Choudhury, R. Corsini, and E. Ciaramella, "3.4 Gbit/s visible optical wireless transmission based on RGB LED," Opt. Exp., vol. 20, no. 26, pp. B501-B506, 2012. 
[4] F.-M. Wu, C.-T. Lin, C.-C. Wei, C.-W. Chen, H.-T. Huang, and C.-H. Ho, "1.1-Gb/s white-LED-based visible light communication employing carrier-less amplitude and phase modulation," IEEE Photon. Technol. Lett., vol. 24, no. 19, pp. 1730-1732, Oct. 1, 2012.

[5] J. M. Kahn and J. R. Barry, "Wireless infrared communications," Proc. IEEE, vol. 85, no. 2, pp. 265-298, Feb. 1997.

[6] J. Fadlullah and M. Kavehrad, "Indoor high-bandwidth optical wireless links for sensor networks," J. Lightw. Technol., vol. 28, no. 21, pp. 30863094, Nov. 1, 2010.

[7] F. R. Gfeller and U. Bapst, "Wireless in-house data communication via diffuse infrared radiation," Proc. IEEE, vol. 67, no. 11, pp. 1474-1486, Nov. 1979.

[8] T. Komine, S. Haruyama, and M. Nakagawa, "Bidirectional visible-light communication using corner cube modulator," in Proc. Wireless and Optical Communication (WOC), Banff, Canada, IASTED, Jul. 24, 2003.

[9] J. Hou and D. O'Brien, "Vertical handover-decision-making algorithm using fuzzy logic for the integrated radio and-OW system," IEEE Trans. Wireless Commun., vol. 5, no. 1, pp. 176-185, Jan. 2006.

[10] Y. Wang, Y. Shao, H. Shang, X. Lu, Y. Wang, J. Yu, and N. Chi, "875-Mb/s asynchronous bi-directional 64QAM-OFDM SCM-WDM transmission over RGB-LED-based visible light communication system," in Proc. Opt. Fiber Commun. Conf. Expo. Nat. Fiber Opt. Eng. Conf., Mar. 17-21, 2013, pp. $1-3$.

[11] K. I. X. Lin and K. Hiroshashi, "High-speed full-duplex multiaccess system for LED based wireless communications using visible light," in Proc Int. Symp. Opt. Eng. Photon. Technol., 2009, pp. 1-5.

[12] G. Cossu, A. M. Khalid, R. Corsini, and E. Ciaramella, "Non-directed line-of-sight visible light system providing high-speed and robustness to ambient light," in Proc. Opt. Fiber Commun. Conf. Expo. Nat. Fiber Opt. Eng. Conf., Mar. 17-21, 2013, pp. 1-3.
[13] A. J. C. Moreira, R. T. Valadas, and A. M. De Oliveira Duarte, "Optical interference produced by artificial light," Wireless Netw., vol. 3, no. 2, pp. 131-140, 1997.

[14] Lighting of Indoor Work Places, European Standard EN, 12464-1, 2003.

[15] A. M. Khalid, G. Cossu, R. Corsini, P. Choudhury, and E. Ciaramella, "1 Gb/s transmission over a phosphorescent white LED by using rateadaptive discrete multitone modulation," IEEE Photon. J., vol. 4, no. 5, pp. 1465-1473, Oct. 2012

[16] Photobiological Safety of Lamps and Lamp Systems, IEC 62471, 2006.

[17] C. Jäger, Eye safety of IREDs Used in Lamp Applications. Application Note. OSRAM Opto Semiconductors, GmbH, Regensburg, Germany, 2009.

[18] R. A. Shafik, S. Rahman, and R. Islam, "On the extended relationships among EVM, BER and SNR as performance metrics," in Proc. Int. Conf. Elect. Comput. Eng., Dec. 19-21, 2006, pp. 408-411.

[19] ITU-T Recommendation, G.975.1, "Forward error correction for high bitrate DWDM submarine systems," Feb. 2004.

[20] C. Duan, G. Pekhteryev, J. Fang, Y. Nakache, J. Zhang, K. Tajima, Y. Nishioka, and H. Hirai, "Transmitting multiple HD video streams over UWB links," in Proc. 3rd IEEE Consum. Commun. Netw. Conf., Jan. 8-10, 2006, vol. 2, pp. 691-695.

Authors' biographies are not available at the time of publication. 